\title{
Long-form census change worries health researchers
}

Published at www.cmaj.ca on July 22

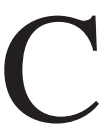

ount health researchers among the many vocal critics of the Conservative government's decision to replace Canada's mandatory long-form census with a voluntary survey. The population data obtained from a voluntary survey will be biased, researchers say, and will inhibit research into the social determinants of health.

The government claims that the long-form census infringes upon the privacy of Canadians, and that citizens should not be compelled to provide personal information under threat of law. These are the reasons the longform census, previously sent to onefifth of Canadian homes every five years, will be replaced by a voluntary survey, which will be sent to one-third of the population. The short-form census, which provides only basic information, remains mandatory.

Academics in various fields - from statistics to economics to health - say the information the long-form census provides is invaluable, and that making it voluntary will result in a dramatically lower response rate and skewed data.

Patricia Martens, director of the Manitoba Centre for Health Policy, says that health researchers were shocked upon learning of the changes to the census. "Everybody who knew about the long-form census and how it's used, their collective jaw dropped simultaneously."

Health researchers link clinical information to population data obtained from the long-form census to determine how socio-economic status affects health. This is done by comparing the rates of disease or other health indicators across various postal codes. Knowing the employment status and average income of people in these postal codes is vital to this type of research. "That information comes from the long-form census," says Martens, who is also a professor in the department of commu-

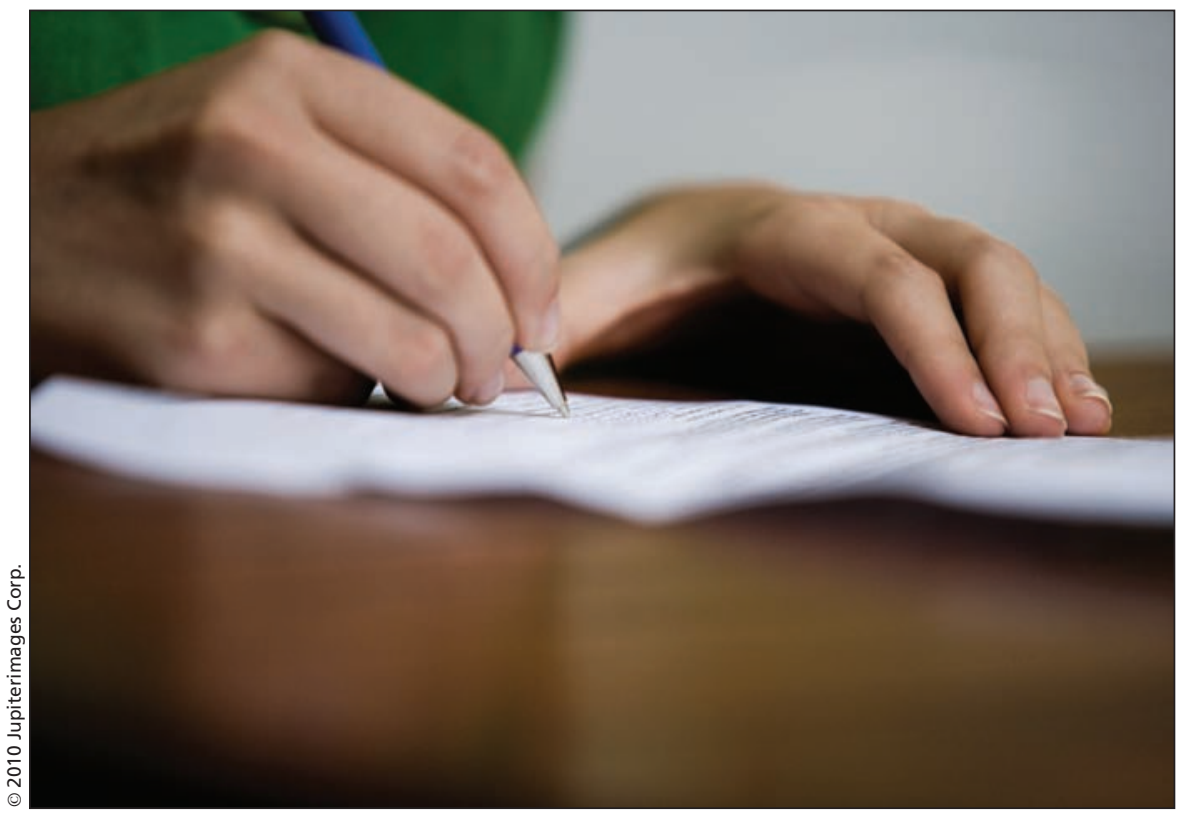

If the long-form census is replaced by a voluntary survey, far fewer people will fill it out and the resulting data will be biased, researchers warn.

nity of health services at the University of Manitoba in Winnipeg. "You can't get it any other way."

According to Martens, research into the social determinants of health is much more than an academic pursuit - it provides evidence that shapes policies intended to lessen health inequities among Canadians in different socio-economic strata. The people who will suffer most if this research is inhibited by inaccurate population data, she says, are those from vulnerable populations, such as First Nations or the poor. Without good data, says Martens, it is difficult to make evidence-based health care decisions at the population level. "It would be quite a tragedy in this era of accountability in decision-making if we don't give decision-makers the tools to be accountable for their decisions."

Experts in statistics say that the information obtained from a voluntary survey differs significantly from that gathered from a mandatory survey. The segment of the population who will complete a voluntary survey will share particular characteristics, as will those who don't complete it, and the resulting sample will therefore not accurately represent the population as a whole. This is a form of sampling bias called "non-response bias."

According to Anne Harris, a PhD candidate in the school of population and public health at the University of British Columbia in Vancouver, research has shown that people at the extremes of the socio-economic scale - the very rich and the very poor are unlikely to fill out voluntary surveys. New immigrants may also be underrepresented, as well as the disabled and people from First Nations communities. Of equal concern to researchers are the subtle biases that a voluntary survey will introduce to census data.

"Those who respond may be very different than those who don't, in ways we don't understand," says Harris. "We are also concerned about the differences we don't know about. You don't want to introduce bias."

Harris is familiar with the debate 
between privacy advocates and public health experts, having explored this topic in her academic career. In a 2008 paper, she looked at the impact that privacy legislation has on health research (Can J Public Health 2008;99:293-6). The negative effects on research, she found, include increases in the cost and duration of studies, study cancellations, decreases in recruitment and study power, decreases in participation rates and the introduction of bias.

"Impediments to health research could result in losses of societal benefits by delaying or prohibiting research into disease etiology, diagnosis and treatment, or health services delivery," the paper concludes. "In the application of public policy, the potential harms of legislation must be weighed against its benefits."

According to Mieke Koehoorn, an associate professor in UBC's school of population and public health, the harms of replacing the long-form census with a voluntary survey do indeed outweigh the benefits. Koehoorn studies occupational and environmental health. In her research, she uses information about rates of injury and disease found in workers' compensation claims, Statis- tics Canada's Canadian Community Health Survey and various provincial sources of health data.

This information, however, must be combined with census data to determine the degree to which different populations are affected by various environmental or occupational health problems. This population data comes from the long-form census. "It's a very powerful tool at the population level," says Koehoorn. "It provides a snapshot of the population that we would never have the resources to collect."

The change to the long-form census may also affect other surveys, including those directly related to health. For instance, the population sample chosen to receive the Canadian Community Health Survey - which Statistics Canada's website describes as providing "cross-sectional estimates of health determinants, health status and health system utilization for 133 health regions across Canada, plus the territories" - is based on census data.

Another major concern among researchers is that data obtained from the new long-form census won't be comparable with data from previous censuses. In an email, Ivan Fellegi, for- mer chief statistician at Statistics Canada, writes that "we will not be able to know whether apparent changes are due to real underlying developments, or are they simply a reflection of the drastic change in methodology between the two censuses. In my mind, this is potentially the most important issue of all!"

Fellegi also disagrees with the government's argument that increasing the number of homes that receive the longform census - at an additional cost of $\$ 30$ million - will compensate for the drop in the participation rate that will result from making it voluntary. "You do not compensate for selective nonresponse by increasing the sample at random; you do not correct for the nonresponding new immigrants or Aboriginal persons by increasing the sample of middle class third generation Canadians," he writes in his email.

The Canadian Institutes of Health Research and the Canadian Institute for Health Information were asked by $C M A J$ how the change to the long-form census will affect health research in Canada, but both organizations declined to comment. — Roger Collier, CMAJ

DOI:10.1503/cmaj.109-3322 